ZASTOSOWANIA MATEMATYKI

APPLICATIONES MATHEMATICAE 20,2 (1990), pp. 281-289

P. TODOROVIC (Santa Barbara, California)

\title{
AN EXTREMAL PROBLEM ARISING IN SOIL EROSION MODELING
}

Under certain conditions the effect of soil erosion on crop production can be described by a random process $\left\{X_{n}\right\}_{1}^{\infty}$, where $X_{n}=Y_{n} L_{n},\left\{Y_{n}\right\}_{1}^{\infty}$ is an i.i.d. sequence of strictly positive r.v.'s, $L_{n}=\prod_{1}^{n} Z_{i}$, and $\left\{Z_{i}\right\}_{1}^{\infty}$ is an i.i.d. sequence of r.v.'s with support $[0,1]$, independent of $\left\{Y_{n}\right\}_{1}^{\infty}$. This paper is concerned with the d.f. of $\chi_{n}=\sup \left\{X_{k} ; 1 \leqslant k \leqslant n\right\}$ and its limit. It was shown that the limiting d.f. is a solution of a Volterra type linear integral equation. The results obtained also include the joint distribution $M_{n}(x, k)$ of $\left(\chi_{n}, N_{n}\right)$, where $N_{n}$ is the "occurrence time" of $\chi_{n}$, as well as the limiting form of this bivariate distribution.

1. Introduction. It seems that one of the first attempts to extend asymptotic results of classical extreme value theory to some classes of dependent r.v.'s was made by Watson [10]. Since that time there have been many further attempts (see, e.g., [1]-[5] and [7]) resulting in generalizations of considerable practical and theoretical interest. A comprehensive account of classical, as well post-classical, results of extreme value theory can be found in [6].

This paper is concerned with some problems of extremal type arising in modeling of the effect of surface soil erosion on crop production. Here we deal with maximum term in a non-stationary sequence of dependent r.v.'s. The results obtained include the joint distribution of the maximum term and its "occurrence time" as well as the limiting form of this bivariate distribution.

2. Preliminaries. In a recent paper [9] it was shown that the effect of soil erosion on crop production may be, under certain conditions, described by a process $\left\{X_{n}\right\}_{1}^{\infty}$, where

$$
X_{n}=Y_{n} L_{n} \quad \text { and } \quad L_{n}=\prod_{i=1}^{n} Z_{i} .
$$

Here $\left\{Y_{n}\right\}_{1}^{\infty}$ and $\left\{Z_{n}\right\}_{1}^{\infty}$ are r.v.'s subject to the following conditions: 
(i) $\left\{Y_{n}\right\}_{1}^{\infty}$ is an i.i.d. sequence of strictly positive r.v.'s with common d.f. $Q(y)=P\{Y \leqslant y\}$ and p.d.f. $q(y)$ such that $\mu=\mathrm{E}\{Y\}<\infty$.

(ii) $\left\{Z_{n}\right\}_{1}^{\infty}$ is also an i.i.d. sequence of r.v.'s with common support in $[0,1]$, independent of $\left\{Y_{n}\right\}_{1}^{\infty}$, such that

$$
H(z)=P\{Z \leqslant z\}, \quad h(z)=H^{\prime}(z) \quad \text { and } \quad \alpha_{1}=\mathrm{E}\{Z\}<1 .
$$

It follows from (ii) that $\left\{L_{n}\right\}_{1}^{\infty}$ is a Markov chain with stationary transition p.d.f.

$$
p(s \mid t)= \begin{cases}t^{-1} h(s), & 0 \leqslant s \leqslant t \leqslant 1 \\ 0 & \text { elsewhere. }\end{cases}
$$

The p.d.f. of $\left(L_{1}, \ldots, L_{n}\right)$ is then

$$
\varphi_{1, \ldots, n}\left(t_{1}, \ldots, t_{n}\right)=h\left(t_{1}\right) \prod_{1}^{n-1} \frac{1}{t_{i}} h\left(\frac{t_{i+1}}{t_{i}}\right) \quad\left(0 \leqslant t_{n} \leqslant \ldots \leqslant t_{1} \leqslant 1\right)
$$

and the p.d.f. of $L_{n}$ is

$$
\varphi_{n}(t)=\int_{t \leqslant t_{n-1} \leqslant \ldots \leqslant t_{1} \leqslant 1} \varphi_{1, \ldots, n}\left(t_{1}, \ldots, t_{n-1}, t\right) \prod_{1}^{n-1} d t_{i} .
$$

Consider

$$
\chi_{n}=\sup _{1 \leqslant j \leqslant n} X_{j}
$$

and denote by

$$
N_{n}=\sum_{k=1}^{n} k I \bigcap_{j \neq k}\left\{X_{k}>X_{j}\right\}
$$

the "time" when $\chi_{n}$ is attained. In this paper we are interested in the d.f.

$$
M_{n}(x)=P\left\{\chi_{n} \leqslant x\right\}
$$

and in its limit as $n \rightarrow \infty$. We shall also determine the joint distribution of $\left(\chi_{n}, N_{n}\right)$ specified as

$$
M_{n}(x, k)=P\left\{\chi_{n} \leqslant x, N_{n}=k\right\}, \quad 1 \leqslant k \leqslant n,
$$

and discuss its asymptotic behaviour as $n \rightarrow \infty$.

3. Some properties of $\left\{\chi_{n}\right\}_{1}^{\infty}$. From conditions (i), (ii) and equation (2.3) we infer at once that

$$
M_{n}(x)=\int_{0 \leqslant t_{n} \leqslant \ldots \leqslant t_{1} \leqslant 1} h\left(t_{1}\right)\left\{\prod_{1}^{n-1} \frac{1}{t_{i}} h\left(\frac{t_{i+1}}{t_{i}}\right)\right\} \prod_{1}^{n} Q\left(\frac{x}{t_{j}}\right) d t_{j} .
$$

The existence of the limit of (3.1) as $n \rightarrow \infty$ is easy to establish: As we have seen in [9], the sum

$$
S=\sum_{1}^{\infty} X_{n}
$$


is an (a.e.) finite-valued r.v. Since $\left\{\chi_{n}\right\}_{1}^{\infty}$ is non-decreasing and dominated by $S$, i.e., $\chi_{n} \leqslant S$ for all $n=1,2, \ldots$, it is clear that $\chi_{n} \uparrow \chi$ (a.e.), where $\chi \leqslant S$ implies that $\chi$ is also (a.e.) finite-valued r.v. Write

$$
M(x)=P\{\chi \leqslant x\}
$$

then from the previous it follows that at least

$$
M_{n}(x) \stackrel{\text { w }}{\rightarrow} M(x) .
$$

Proposition 3.1. The d.f. $M(x)$ is a solution of the following Volterra singular linear integral equation:

$$
M(x)=x \int_{x}^{\infty} \frac{1}{u^{2}} h\left(\frac{x}{u}\right) Q(u) M(u) d u .
$$

Proof. From the definition of $\chi$ we obtain

where

$$
\begin{aligned}
\chi & =\sup \left\{Y_{1} L_{1}, Y_{2} L_{2}, \ldots\right\} \\
& =\sup \left\{Y_{1}, Y_{2} Z_{1}, Y_{3} Z_{2} Z_{3}, \ldots\right\} Z_{1}=\sup \left\{Y_{1}, \chi^{*}\right\} Z_{1},
\end{aligned}
$$

$$
\chi^{*}=\sup \left\{Y_{2} Z_{2}, Y_{3} Z_{2} Z_{3}, \ldots\right\} .
$$

It is clear from conditions (i) and (ii) that $\chi^{*}$ is a version of $\chi$ independent of $\left(Y_{1}, Z_{1}\right)$. This and (3.4) yield at once that

$$
M(x)=\int_{0}^{1} Q\left(\frac{x}{s}\right) M\left(\frac{x}{s}\right) h(s) d s,
$$

from which, after a simple transformation, the proposition follows. theorem:

In view of this result, we can formulate the following characterization.

Corollary 3.1. Let $Z$ with support in $[0,1]$ and $P\{Z=1\}<1$, and $Y>0$ (a.e.) with $\mathrm{E}\{Y\}<\infty$ be two independent r.v.'s. Then there exists a unique distribution with support in $(0, \infty)$, such that if $\chi$ has this distribution and is. independent of $Z$ and $Y$, then

$$
\chi \stackrel{d}{=} \max \{Y, \chi\} Z \text {. }
$$

Remark 3.1. We can relax somewhat regularity restrictions (i) and (ii) without changing substantially Proposition 3.1. Suppose for instance that $\left\{\left(Z_{n}, Y_{n}\right)\right\}_{1}^{\infty}$ is an i.i.d. sequence with common probability density $f(s, y)$; then in (3.4) $\chi^{*}$ is still independent of $\left(Z_{1}, Y_{1}\right)$ and

$$
\chi \stackrel{\mathrm{d}}{=} \chi^{*} \text {. }
$$


Therefore from (3.4) it follows at once that

where

$$
M(x)=\int_{0}^{1} F\left(\frac{x}{s} \mid s\right) M\left(\frac{x}{s}\right) d s,
$$

Write

$$
h(s)=\int_{0}^{\infty} f(s, y) d y \quad \text { and } \quad F(y \mid s)=\int_{0}^{y} f(u \mid s) d u .
$$

and denote by

$$
\chi_{k, n}=\sup _{k \leqslant j \leqslant n} X_{j}, \quad k=1, \ldots, n,
$$

$$
M_{k, n}(x)=P\left\{\chi_{k, n} \leqslant x\right\}
$$

the corresponding d.f. The next result is a generalization of Proposition 3.1.

Proposition 3.2. For every $k=1,2, \ldots$,

$$
M_{(k)}(x)=\int_{0}^{1} Q\left(\frac{x}{s}\right) M\left(\frac{x}{s}\right) \varphi_{k}(s) d s,
$$

where $M_{k, n}(x) \stackrel{w}{\rightarrow} M_{(k)}(x)$ as $n \rightarrow \infty$ and $\varphi_{k}(s)$ is given by (2.4).

Proof. Since

$$
\chi_{k, n}=\sup \left\{Y_{k} L_{k}, \ldots, Y_{n} L_{n}\right\}=\sup \left\{Y_{k}, \chi_{n-k}^{*}\right\} L_{k},
$$

where $\chi_{n-k}^{*}$ is a version of $\chi_{n-k}$, independent of $\left(Y_{k}, L_{k}\right)$. This gives at once the following:

$$
M_{k, n}(x)=\int_{0}^{1} Q\left(\frac{x}{s}\right) M_{n-k}\left(\frac{x}{s}\right) \varphi_{k}(s) d s .
$$

From this, invoking Proposition 3.1 and the Beppo-Levi theorem, the proposition follows.

4. A case study. A mapping $f: R^{(n)} \rightarrow R$ is said to be completely symmetric if it is invariant with respect to permutations of its variables. If the mapping is invariant with respect to permutations of some of its variables, we shall say that $f$ is partially symmetric.

Let a function $f(\cdot, \ldots, \cdot), R$-integrable on the $n$-dimensional cube $(a<b)$,

$$
C_{n}(a, b)=\left\{X_{(n)} ; a \leqslant x_{i} \leqslant b ; i=1, \ldots, n\right\},
$$

be partially symmetric with respcet to its first $k$ variables; then we have

$$
\begin{aligned}
& \quad \int_{a \leqslant x_{n} \leqslant \ldots \leqslant x_{1} \leqslant b} f\left(x_{1}, \ldots, x_{n}\right) \prod_{1}^{n} d x_{i} \\
& =\frac{1}{k !} \int_{a \leqslant x_{n} \leqslant \ldots \leqslant x_{k+1} \leqslant b}\left\{\int_{x_{k+1}}^{b} \ldots \int_{x_{k+1}}^{b} f\left(u_{1}, \ldots, u_{k}, x_{k+1}, \ldots, x_{n}\right) \prod_{1}^{k} d u_{i}\right\} \prod_{k+1}^{n} d x_{j} .
\end{aligned}
$$


For a proof of this and more details see [8].

It was pointed out in [9] that a plausible distribution of the r.v. $Z$ may be the following one:

$$
h(s)=\alpha s^{\alpha-1}
$$

for some $\alpha \geqslant 1$. In such a case it follows from (3.1) that

$$
\begin{aligned}
M_{n}(x) & =\alpha^{n} \int_{0 \leqslant t_{n} \leqslant \ldots \leqslant t_{1} \leqslant 1} \frac{\left(t_{1} \ldots t_{n}\right)^{\alpha-1}}{\left(t_{1} \ldots t_{n-1}\right)^{\alpha}} \prod_{1}^{n} Q\left(\frac{x}{t_{j}}\right) d t_{j} \\
& =\alpha^{n} \int_{0 \leqslant t_{n} \leqslant \ldots \leqslant t_{1} \leqslant 1}\left\{\prod_{1} \ldots \frac{1}{t_{j}} Q\left(\frac{x}{t_{j}}\right) d t_{j}\right\} t_{n}^{\alpha-1} Q\left(\frac{x}{t_{n}}\right) d t_{j} .
\end{aligned}
$$

Now, the integrand is partially symmetric with respect to $\left(t_{1}, \ldots, t_{n-1}\right)$, so that according to (4.1) we have

$$
M_{n}(x)=\frac{\alpha^{n}}{(n-1) !} \int_{0}^{1}\left\{\int_{t}^{1} \frac{1}{s} Q\left(\frac{x}{s}\right) d s\right\}^{n-1} t^{\alpha-1} d t
$$

Since for $n \geqslant 2$

$$
t^{\alpha-1}\left\{\int_{t}^{1} \frac{1}{s} Q\left(\frac{x}{s}\right) d s\right\}^{n-1} Q\left(\frac{x}{t}\right)=-\frac{t^{\alpha}}{n} \frac{d}{d t}\left\{\int_{t}^{1} \frac{1}{s} Q\left(\frac{x}{s}\right) d s\right\}^{n},
$$

integration by parts of (4.3) gives for $n \geqslant 2$

$$
M_{n}(x)=\frac{\alpha^{n+1}}{n !} \int_{0}^{1}\left\{\int_{t}^{1} \frac{1}{s} Q\left(\frac{x}{s}\right) d s\right\}^{n} t^{\alpha-1} d t
$$

and

$$
M_{1}(x)=\alpha \int_{0}^{1} s^{\alpha-1} Q\left(\frac{x}{s}\right) d s
$$

The limit of (4.4) as $n \rightarrow \infty$ can be obtained as a solution of the integral equation (3.3). Substituting (4.2) in (3.3) we have

$$
M(x)=\alpha x^{\alpha} \int_{x}^{\infty} \frac{1}{u^{\alpha+1}} Q(u) M(u) d u .
$$

Differentiating both sides of this equation we get

$$
x M^{\prime}(x)=\alpha\{1-Q(x)\} M(x) .
$$

From this, taking into account that $M(x) \rightarrow 1$ as $x \rightarrow \infty$, it follows that

$$
M(x)=\exp \left\{-\alpha \int_{x}^{\infty} u^{-1}[1-Q(u)] d u\right\} .
$$

Remark 4.1. One can obtain (4.5) directly from (4.4) by showing that the d.f. $M_{n}(x)$ satisfies the following difference-differential equation: 


$$
M_{n}^{\prime}(x)=\frac{\alpha}{x}\left\{M_{n}(x)-Q(x) M_{n-1}(x)\right\}
$$

and then letting $n \rightarrow \infty$.

Remark 4.2. Due to the fact that (4.7) is continuous, the convergence of (4.3) is uniform.

5. On the limit of $M_{n}(x, k)$. Consider the sequence of r.v.'s $\left\{N_{n}\right\}_{1}^{\infty}$ defined by (2.6). Since, for every $k=2, \ldots, n$,

$$
\left\{N_{n} \geqslant k\right\}=\left\{\chi_{k-1} \leqslant \chi_{k, n}\right\},
$$

it follows that

$$
N_{n}=1+\sum_{k=2}^{n} I_{\left\{N_{n} \geqslant k\right\}}=1+\sum_{k=2}^{n} I_{\left\{\chi_{k-1} \leqslant \chi_{k}, n\right\}}
$$

From this we see clearly that $\left\{N_{n}\right\}_{1}^{\infty}$ is non-decreasing and as such

$$
N_{n} \uparrow N \text { (a.e.) as } n \rightarrow \infty \text {. }
$$

We want to show that the r.v. $N$ is (a.e.) finite valued. To this end notice that, for all $i=2,3, \ldots$,

$$
\{N \leqslant i\}=\left\{\chi_{i} \geqslant \chi_{(i+1)}\right\} .
$$

The rest of the proof depends on the following observation: for every $k=1,2, \ldots$,

$$
\chi_{(k+1)}=L_{k} \chi_{0}^{*}
$$

where $\chi_{0}^{*}$ is a version of $\chi$ independent of $\left(L_{k}, \chi_{k}\right)$. As we know (see [9]) $L_{k} \rightarrow 0$ (a.e.) as $k \rightarrow \infty$, and thus $\chi_{(k)} \rightarrow 0$ (a.e.) as $k \rightarrow \infty$. Hence

$$
P\{N<\infty\}=\lim _{i \rightarrow \infty} P\{N \leqslant i\}=\lim _{i \rightarrow \infty} P\left\{\chi_{i} \geqslant \chi_{(i+1)}\right\}=1
$$

and the assertion follows.

Let $M(x, k)$ be the distribution of $(\chi, N)$ defined as

$$
M(x, k)=P\{\chi \leqslant x, N=k\}, \quad k=1,2, \ldots
$$

Since $\left(\chi_{n}, N_{n}\right) \uparrow(\chi, N)$ (a.e.), it follows that at least

$$
M_{n}(x, k) \stackrel{w}{\rightarrow} M(x, k) .
$$

In this section we shall determine (5.4). To this end we need the next lemma. Write

$$
G_{k}(s, x)=P\left\{L_{k} \leqslant s, \chi_{k} \leqslant x\right\}
$$

then we have

LEMMA 5.1. For every $k=1,2, \ldots$, 


$$
G_{k}(s, x)=\int_{0 \leqslant t_{k} \leqslant \ldots \leqslant t_{1} \leqslant s} h\left(t_{1}\right)\left\{\prod_{1}^{k-1} \frac{1}{t_{i}} h\left(\frac{t_{i+1}}{t_{i}}\right)\right\} \prod_{1}^{k} Q\left(\frac{x}{t_{j}}\right) d t_{j} .
$$

Proof. It is straightforward and direct that

$$
\begin{aligned}
P\left\{L_{k} \leqslant s, \chi_{k} \leqslant x\right\} & =P\left\{L_{k} \leqslant s, Y_{1} L_{1} \leqslant x, \ldots, Y_{k} L_{k} \leqslant x\right\} \\
& =\int_{s \leqslant t_{k} \leqslant \ldots \leqslant t_{1} \leqslant 1} \varphi_{1, \ldots, k}\left(t_{1}, \ldots, t_{k}\right) \prod_{1}^{k} Q\left(\frac{x}{t_{j}}\right) d t_{j} .
\end{aligned}
$$

This and (2.3) prove the assertion.

It is clear from (5.6) that the p.d.f. $g_{k}(s, x)$ of $\left(L_{k}, \chi_{k}\right)$ exists. We now have

Proposition 5.1. For every $k=2,3, \ldots$,

$$
M(x, k)=\int_{0}^{x} \int_{0}^{1} M(u / s)\left\{g_{k}(s, u)-g_{k-1}(s, u)\right\} d s d u
$$

and

$$
M(x, 1)=\int_{0}^{x} \int_{0}^{1} M(u / s) g_{1}(s, u) d s d u
$$

Proof. Our proof is based on the following relation: For every $k=1,2, \ldots$ and $x \geqslant 0$,

$$
P\{\chi \leqslant x, N \leqslant k\}=P\left\{\chi_{k} \leqslant x, \chi_{k} \geqslant \chi_{(k+1)}\right\} .
$$

From this we have

$$
\begin{aligned}
& M(x, 1)=P\left\{X_{1} \leqslant x, X_{1} \geqslant \chi_{(2)}\right\}, \\
& M(x, k)=P\left\{\chi_{k} \leqslant x, \chi_{k} \geqslant \chi_{(k+1)}\right\}-P\left\{\chi_{k-1} \leqslant x ; \chi_{k-1} \geqslant \chi_{(k)}\right\} .
\end{aligned}
$$

Now, taking into account equation (5.3) we see at once that

$$
P\left\{\chi_{k} \leqslant x, \chi_{k} \geqslant \chi_{(k+1)}\right\}=\int_{0}^{x} \int_{0}^{1} M(u / s) g_{k}(s, u) d s d u,
$$

which proves the assertion.

A general form of the distribution $M_{n}(x, k)$ has a very cumbersome expression. A somewhat simplified version is given by the following proposition:

Proposition 5.2. For $n \geqslant 2$,

$$
\begin{aligned}
& M_{n}(x, 1)=\int_{0}^{x} \int_{0}^{1} M_{n-1}(u / s) g_{1}(s, u) d s d u \\
& M_{n}(s, k)=\int_{0}^{x} \int_{0}^{1}\left\{M_{n-k}(u / s) g_{k}(s, u)-M_{n-k+1}(u / s) g_{k-1}(s, u)\right\} d s d u
\end{aligned}
$$

for $2 \leqslant k \leqslant n-1$, and 


$$
M_{n}(x, n)=M_{n}(x)-\int_{0}^{x} \int_{0}^{1} M_{1}(u / s) g_{n-1}(s, u) d s d u \text {. }
$$

Proof. We observe that the following holds for all $k=1, \ldots, n-1$ :

$$
P\left\{\chi_{n} \leqslant x, N_{n} \leqslant k\right\}=P\left\{\chi_{k} \leqslant x, \chi_{k} \geqslant \chi_{k+1, n}\right\} .
$$

In addition,

$$
\chi_{k+1, n} \stackrel{d}{=} L_{k} \chi_{n-k}^{*}
$$

where $\chi_{n-k}^{*}$ is a version of $\chi_{n-k}$ independent of $\chi_{n-k}$. From this, after some straightforward calculations, the assertion follows.

Remark 5.1. Since $X_{n} \rightarrow 0$ (a.e.), we infer from the equality

$$
M_{n}(x, n)=P\left\{X_{n} \leqslant x, \chi_{n-1} \leqslant X_{n}\right\}
$$

that $M_{n}(x, n) \rightarrow 0$ as $n \rightarrow \infty$ uniformly in $x$. Now letting $n \rightarrow \infty$ in Proposition 5.2 we obtain, taking into account the Beppo-Levi theorem, yet another proof of Proposition 5.1.

6. An application. We shall outline a possible application of some of the previous results. As we have seen in [9], in the presence of soil degradation due to erosion, we are concerned with the duration of economically viable productivity period. The question is how long, under steady soil erosion, an economically effective crop production shall be maintained? We begin our discussion by establishing certain criteria for an economic production.

The following definition, which represents a somewhat oversimplified view of economic productivity, can be used as a first approximation. We assume that the crop production is economically viable as long as it can be maintained above a certain threshold level $x_{0}$. Let $D\left(x_{0}\right)$ be the duration of an economically viable productivity period, i.e.

$$
D\left(x_{0}\right)=\sup \left\{n ; X_{n}>x_{0}\right\} .
$$

It is clear from (6.1) that

$$
\left\{D\left(x_{0}\right)=0\right\}=\left\{\chi \leqslant x_{0}\right\}
$$

and that

Hence

$$
\left\{D\left(x_{0}\right)=k\right\}=\left\{X_{k}>x_{0}, \chi_{(k+1)} \leqslant x_{0}\right\}=\left\{\chi_{(k+1)} \leqslant x_{0}<\chi_{(k)}\right\} .
$$

$$
\begin{aligned}
& P\left\{D\left(x_{0}\right)=0\right\}=M\left(x_{0}\right), \\
& P\left\{D\left(x_{0}\right)=k\right\}=M_{(k+1)}\left(x_{0}\right)-M_{(k)}\left(x_{0}\right)
\end{aligned}
$$

for $k=1,2, \ldots$ Since $L_{k} \rightarrow 0$ (a.e.), it follows from (5.11) that

$$
P\left\{D\left(x_{0}\right)<\infty\right\}=1 \quad \text { for every } x_{0}>0 .
$$


It is also easy to show that

$$
\mathrm{E}\left\{D\left(x_{0}\right)\right\}=\sum_{k=1}^{\infty} \int_{0}^{1}\left\{1-Q\left(x_{0} / s\right) M\left(x_{0} / s\right)\right\} \varphi_{k}(s) d s .
$$

In the particular case where $h(s)=\alpha s^{\alpha-1}$ we obtain from (2.3), (2.4) and (4.1) that

$$
\varphi_{k}(s)=\frac{\alpha^{k}}{\Gamma(k)} s^{\alpha-1}(-\ln s)^{k-1} \quad \text { for every } k=1,2, \ldots
$$

This and (3.7) yield, after some straightforward calculations, that

$$
M_{k}(x)=\frac{(\alpha x)^{k}}{\Gamma(k)} \int_{x}^{\infty} \frac{(\ln u / x)^{k-1}}{u^{\alpha+1}} Q(u) \exp \left\{-\alpha \int_{u}^{\infty} s^{-1}[1-Q(s)] d s\right\} d u \text {. }
$$

\section{References}

[1] S. M. Berman, Limiting distribution of the maximum term in sequences of dependent random variables, Ann. Math. Statist. 33 (1962), pp. 894-908.

[2] - Limit theorems for the maximum term in stationary sequences, ibidem 35 (1964), pp. 502-516.

[3] - Sojourns and extremes of stationary processes, Ann. Probab. 10 (1982), pp. 1-46.

[4] H. Cramér, On the maximum of a normal stationary stochastic process, Bull. Amer. Math. Soc. 68 (1962), pp. 512-516.

[5] R. M. Leadbetter, On extreme value in stationary sequences, Z. Wahrsch. Verw. Gebiete 28 (1974), pp. 289-303.

[6] - G. Lindgren and H. Rootzen, Extremes and Related Properties of Random Sequences and Processes, Springer-Verlag, New York 1983.

[7] R. M. Loy nes, Extreme values in uniformly mixing stationary process, Ann. Math. Statist. 36 (1965), pp. 993-999.

[8] P. Todorovic, An extremal problem, Technical Report No. 240, Dept. of Statistics, University of Kentucky.

[9] - and J. Gani, Modeling of the effect of erosion on crop production, J. Appl. Probab. 24 (1987), pp. 787-797.

[10] G. S. W ats on, Extreme values in samples from m-dependent stationary stochastic processes, Ann. Math. Statist. 25 (1954), pp. 798-800.

DEPARTMENT OF MATHEMATICS

PROGRAM IN STATISTICS AND

APPLIED PROBABILITY

UNIVERSITY OF CALIFORNIA

SANTA BARBARA, CA 93106
U.S.A.

Received on 1987.04.28;

revised version on 1987.10 .05 